\title{
PRESENCIA DE Teredolites EN LA FORMACIÓN ARCILLAS DE MORELLA (CRETÁCICO INFERIOR, CASTELLÓN)
}

\author{
Oriol FERRER ${ }^{1}$ y Jordi M. de GIBERT ${ }^{2}$ \\ ${ }^{1}$ Departament de Geodinàmica i Geofísica, Universitat de Barcelona. Martí i \\ Franquès s/n, 08028 Barcelona. Correo electrónico: joferrer@geo.ub.es \\ ${ }^{2}$ Departament d'Estratigrafia, Paleontologia i Geociències Marines, Universi- \\ tat de Barcelona. Martí i Franquès s/n, 08028 Barcelona. Correo electrónico: \\ jmdegibert@ub.edu
}

Ferrer, O. \& Gibert, J. M. de 2005. Presencia de Teredolites en la Formación Arcillas de Morella (Cretácico Inferior, Castellón). [Presence of Teredolites in the Morella Mudstone Formation (Lower Cretaceous, Castellón).] Revista Española de Paleontología, N.E. X, 39-47. ISSN 0213-6937.

\begin{abstract}
The present paper documents the presence of bored tree logs in the Morella Mudstone Formation (Lower Aptian, Castellón). Two different forms have been recognized corresponding to two different ichnospecies: Teredolites clavatus and Teredolites longissimus. The borings are similar to those produced nowadays by bivalves of the families Pholadidae and Teredinidae. The presence of the borings in the upper part of the Morella Mudstone Formation, together with other sedimentological and paleontological data, evidence a clear marine (tidal) influence for these deposits. The horizon containing Teredolites is interpreted as a transgressive surface (TS) separating the continental red mudstone facies that constitute the lowstand systems tract (LST) and the marine heterolitic facies corresponding to the transgressive systems tract (TST).
\end{abstract}

Keywords: Teredolites, bioerosion, ichnology, Morella Mudstone Formation, Lower Cretaceous.

\section{RESUMEN}

En el presente trabajo se da a conocer la presencia de troncos con perforaciones en la Formación Arcillas de Morella (Aptiense Inferior, Castellón). Se han reconocido dos formas correspondientes a dos icnoespecies diferentes: Teredolites clavatus y Teredolites longissimus. Las perforaciones se asignan a la actividad perforante de bivalvos de las familias Pholadidae y Teredinidae. La presencia de estas perforaciones en los niveles superiores de la Formación Arcillas de Morella, junto con el contenido paleontológico asociado a éstas, así como las estructuras sedimentarias de los diferentes materiales, confirman que estos depósitos poseen una clara influencia mareal. La base del nivel con Teredolites corresponde a una superficie transgresiva (TS) que separaría los depósitos continentales del cortejo de bajo nivel del mar (LST) caracterizado por las facies de arcillas rojas de la mitad inferior de la unidad, de los del cortejo transgresivo (TST), constituido por las facies heterolítico-arenosas de la parte superior de esta formación, de clara influencia mareal.

Palabras clave: Teredolites, bioerosión, icnología, Formación Arcillas de Morella, Cretácico Inferior.

\section{INTRODUCCIÓN}

En una serie de trabajos de campo realizados en los alrededores de la localidad de Morella (en el noroeste de la provincia de Castellón) se levantaron diversos perfi les estratigráfi cos en la Formación Arcillas de Morella. Esta formación conocida desde hace mucho tiempo sobre todo por la abundancia de restos de dinosaurios (Beltrán, 1918) ha sido estudiada por numerosos autores en el ámbito es- tratigráfico (Canerot et al., 1982; Salas \& Martín-Closas, 1995; Salas \& Guimerà, 1997; Salas et al., 2001; Gámez et al., 2003) y paleontológico (Santafé et al., 1978, 1981, 1982; Ruiz Omeñaca \& Santos Cubedo, 1998). La presente contribución documenta la presencia de perforaciones de bivalvos en troncos, atribuibles al icnogénero Teredolites Leymerie, 1842, en los niveles más altos de dicha formación. El horizonte de donde provienen las perforaciones es interpretado como correspondiente a las facies 
de abandono de un sistema deltaico, desarrollado durante la regresión marina del Aptiense inferior en la cuenca del Maestrat (Salas \& Martín-Closas, 1995).

\section{CONTEXTO GEOLÓGICO Y ESTRATIGRÁFICO}

La localidad de Morella se sitúa al noroeste de la provincia de Castellón en la comarca de Els Ports. Desde un punto de vista geológico, la zona se incluye en la denominada subcuenca de Morella, situada en el sector noroccidental de la cuenca del Maestrat (Fig. 1 a). La Formación Arcillas de Morella, del Cretácico Inferior, presenta un gran desarrollo en la cuenca del Maestrat (Fig. 1 b), en donde puede alcanzar los 100 metros de potencia (Fig. 2), aunque se extiende también a las vecinas cuencas de Galve y Penyagolosa. Ambas cuencas forman parte de las denominadas cuencas mesozoicas intracontinentales del margen oriental de Iberia (Salas \& Martín-Closas, 1995) y poseen una historia geológica compleja que comprende entre otros eventos tectónicos dos ciclos de rift.

La Formación Arcillas de Morella se incluye dentro del ciclo Jurásico Superior-Cretácico Inferior, durante el cual un sistema de fallas normales estructuró la cuenca en siete subcuencas principales (Salas \& Guimerà, 1996). En este contexto se depositó la megasecuencia cretácica inferior, formada por al menos 10 secuencias de depósito (Salas \& Martín-Closas, 1995). La Formación Arcillas de Morella quedaría comprendida dentro de la secuencia de depósito del Aptiense inferior (K1.8 de Salas \& MartínClosas, 1995) (Fig. 2). Dentro de esta secuencia se delimitan cinco unidades litoestratigráficas que comprenden una potencia total de 800 metros. Las más inferiores son las formaciones Morella y Cervera. Estas unidades se superponen a las plataformas barremienses de la Formación
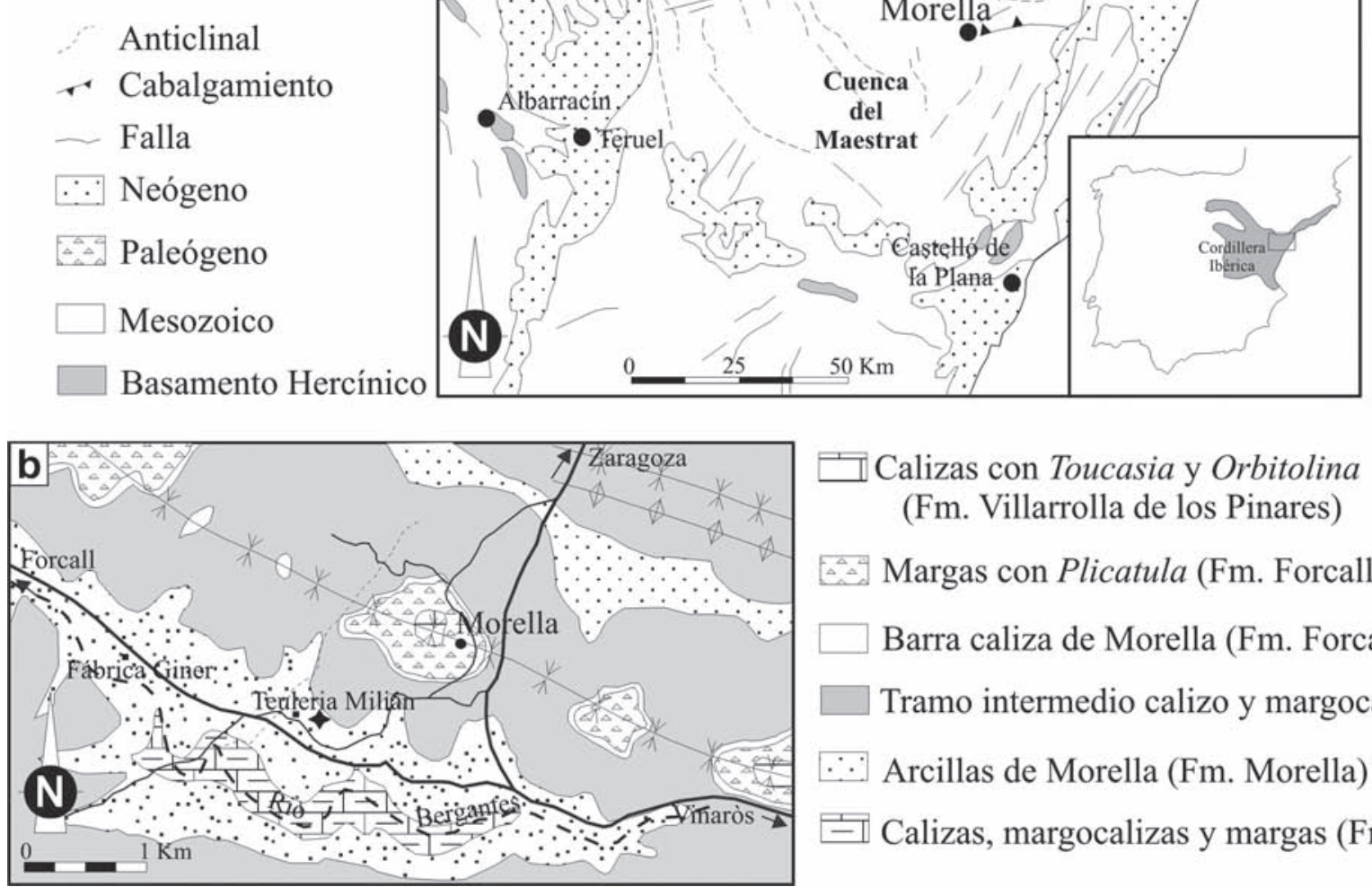

Calizas con Toucasia y Orbitolina (Fm. Villarrolla de los Pinares)

Margas con Plicatula (Fm. Forcall)

Barra caliza de Morella (Fm. Forcall)

Tramo intermedio calizo y margocalizo (Fm. Xert)

Arcillas de Morella (Fm. Morella)

Calizas, margocalizas y margas (Fm. Cervera)

Figura 1. a, Mapa estructural simplificado de la Cuenca del Maestrat (modificado de Salas \& Martín-Closas, 1995). b, Mapa geológico simplificado de los alrededores de la localidad de Morella con la situación del perfil levantado en la Teuleria Milian (modificado de IGME, 1973).

$\boldsymbol{a}$, Synthetic structural map of the Maestrat Basin (modified from Salas \& Martin-Closas, 1995). b, Synthetic geological map of the Morella area with indication of the situation of the Teuleria Milian (modified from IGME, 1973). 
Artoles que constituye el techo de la secuencia K1.7. La relación estratigráfica entre las Arcillas de Morella y las Margas de Cervera es de cambio lateral de facies. Ambas

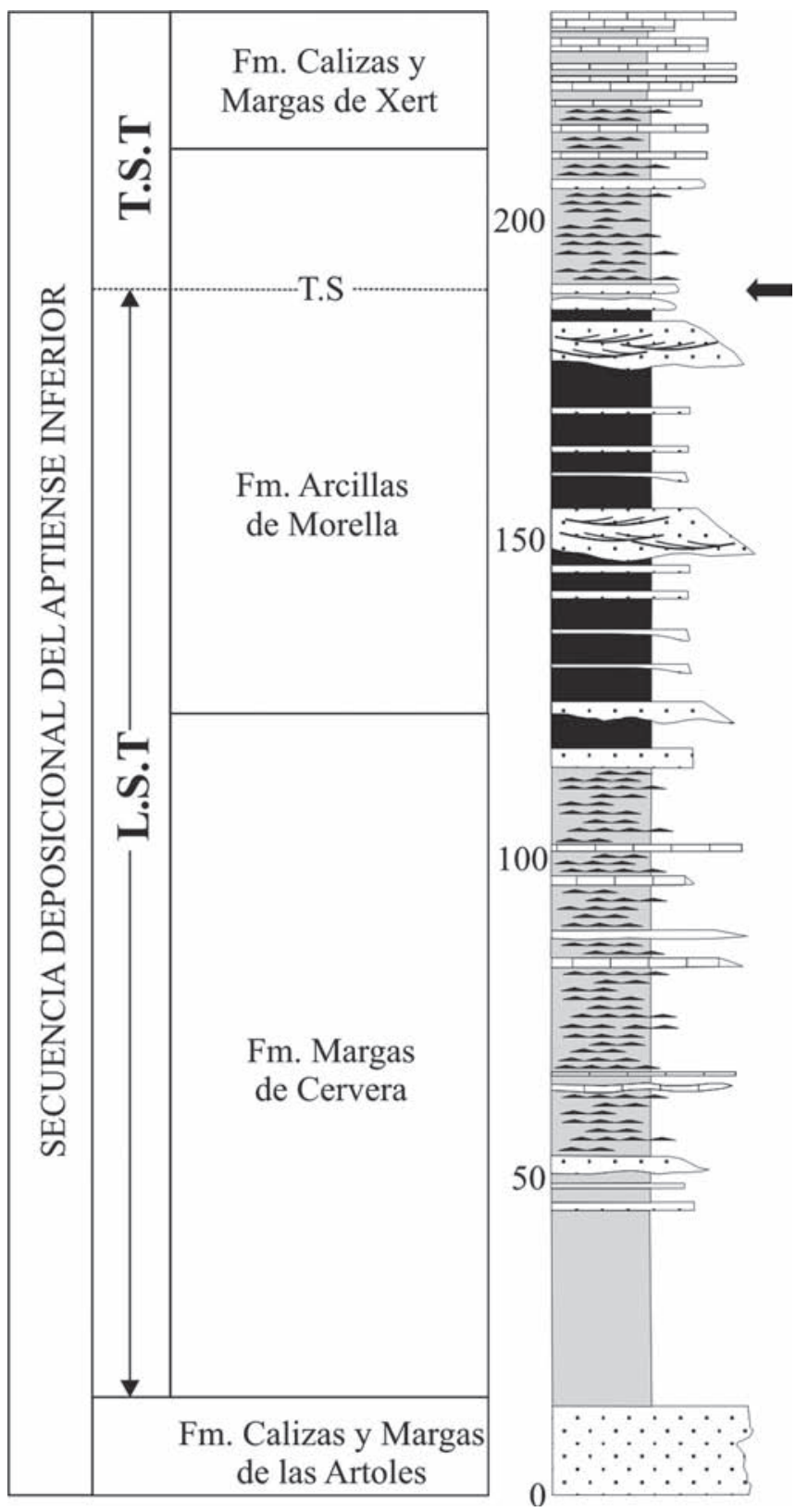

Figura 2. Columna estratigráfica sintética de la parte inferior de la secuencia deposicional del Aptiense (K1.8) (modificado de Salas \& Martín Closas, 1995). La flecha indica el nivel con Teredolites Leymerie, 1842 en el tramo superior de las Arcillas de Morella (escala de la figura en metros).

Synthetic stratigraphic section of the lower part of the Aptian (K1.8) depositional sequence (modified from Salas \& Martín-Closas, 1995). The arrow indicates the horizon with Teredolites Leymerie, 1842 in the upper part of the Morella mudstones (scale in meters). representan una importante entrada de sedimentos terrígenos interpretada, en términos de estratigrafía secuencial, como el cortejo de bajo nivel del mar (Salas \& MartínClosas, 1995). El ascenso del nivel del mar se empieza a detectar hacia techo de la Formación Arcillas de Morella que junto con las formaciones Xert y Forcall constituyen el cortejo transgresivo. El cortejo de alto nivel del mar coincidiría con la sedimentación de la base de la Formación Villarroya de los Pinares constituida por una barra de Toucasias (Salas \& Martín-Closas, 1995).

\section{ESTRATIGRAFÍA Y PALEONTOLOGÍA DEL PERFIL DE LA TEULERIA MILIÁN}

El material estudiado procede de los niveles más altos de la Formación Arcillas de Morella en la explotación conocida como Teuleria Milian situada a $1,5 \mathrm{~km}$ al SO de la localidad de Morella (Fig. 1 b). De forma general, el perfil levantado (Fig. 3 a) está formado de base a techo por una unidad poco potente de calcarenitas con ostreidos (Ceratostreon tuberculiferum Koch \& Dunker, 1837) que dan paso a limolitas arcillosas de color rojo vino que constituyen la mayor parte de la formación. Intercalados entre las arcillas se identifican algunos niveles de areniscas grises de granulometría fina a media con estructuras de bioturbación atribuidos a los icnogéneros Skolithos Haldeman, 1840 y Thalassinoides Ehrenberg, 1944. Interestratificados en las arcillas rojas también se observan numerosos paquetes de areniscas ocres con lags basales ricos en restos vegetales y cantos blandos. Estas areniscas presentan numerosas estructuras sedimentarias entre las que destacan estratificación cruzada planar, estratificación cruzada en surco y de bajo ángulo y estructuras de deformación. Hacia la parte superior de dicha formación (a partir del metro 64 del perfil medido, Fig. 3 a) se produce un marcado cambio de las facies dominantemente limolítico-arcillosas de color rojo a facies heterolítico-arenosas de colores grisáceos. Es sobre el contacto entre estos dos conjuntos de facies donde se han localizado los troncos con perforaciones asignables a Teredolites Leymerie, 1842 en la Teuleria Milian. Los niveles arenosos de las facies heterolíticas presentan estructuras sedimentarias como estratificación y laminación cruzada en surco, ripples de corriente y tidal boundlets.

Gámez et al. (2003) y Salas et al. (2003) estudiaron la Formación Arcillas de Morella en el Mas de la Parreta, situado a $1,5 \mathrm{~km}$ de la Teuleria Milian, y reconocieron cinco asociaciones de facies a las que asignan diversos ambientes sedimentarios. Estas asociaciones son también reconocibles en la Teuleria Milian. Así los niveles basales carbonatados corresponden a ambientes sedimentarios de llanura mareal carbonatada (asociación 1). Por encima de ellos, se desarrollaría una llanura fangosa (mud flat) que estaría caracterizada por las limolitas arcillosas rojizas 


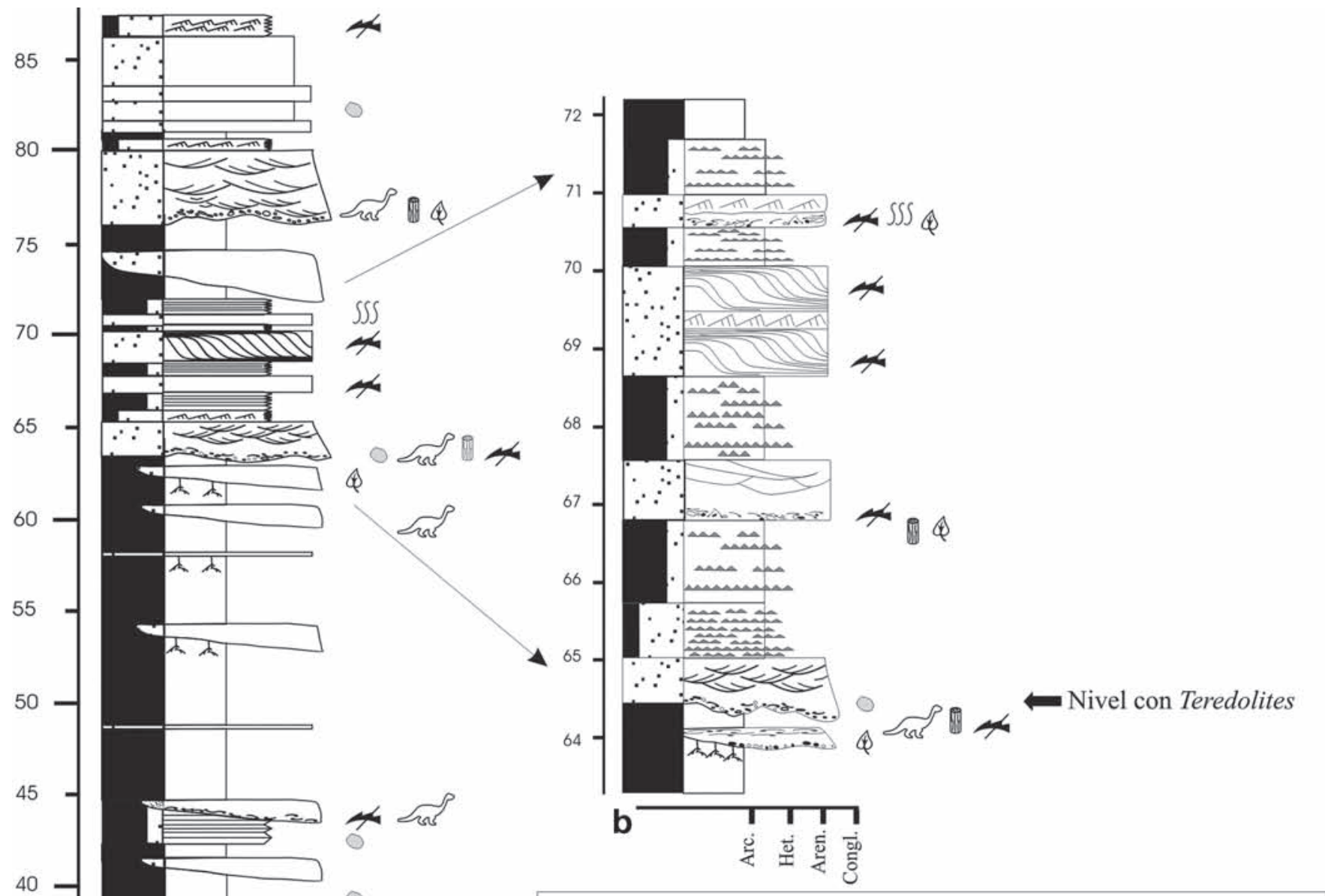

Leyenda:

30

25

20

10

.

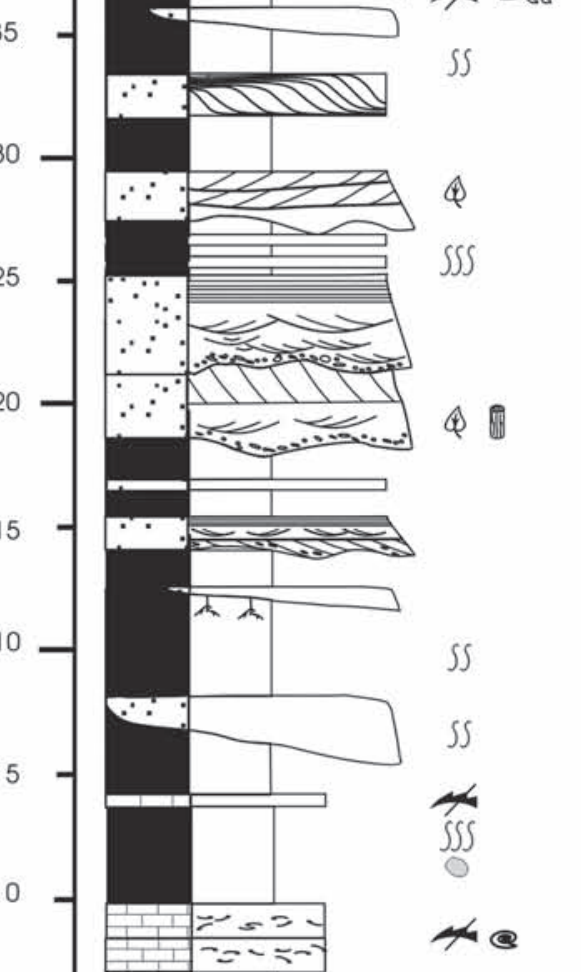

Litologías:

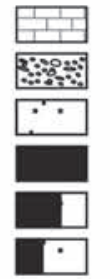

Calizas Conglomerados Areniscas

Arcillas

Heterolítico arcillosa

Heterolítico arenosa

Estructuras sedimentarias

Contenido paleontológico

$\equiv$ Laminación paralela

Facies heteroliticas

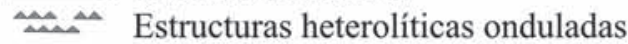

Estratificación cruzada de surco

$\sum$ Estratificación cruzada planar

Tidal Bundles

man Ripples de corriente

$\rightleftharpoons$ Superficies de reactivación

$\longrightarrow$ Cuerpos lenticulares

_ Base erosiva

- Nódulos ferruginosos

$\therefore \quad$ Intraclastos

$\therefore$ Extraclastos
Vertebrados

Q Plantas

Troncos

4 Ostreidos fragmentados

Q Gasterópodos

1. Marcas de raíces

SS Bioturbación media

$\iint S$ Bioturbación elevada

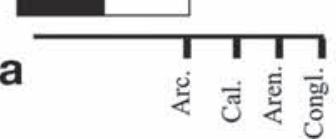


(asociación 2). Esta llanura estaría cruzada por canales de diferente jerarquía con influencias fluviales o mareales (asociaciones 3 y 4). Finalmente hacia la parte superior, se desarrolló una llanura siliciclástica mareal (asociación 5) en la que probablemente también se desarrollaron ambientes de playa.

En mayor detalle, los niveles en los que se encuentran los troncos perforados en la Teuleria Milian (Fig. 3 b), están caracterizados por una asociación de facies formada de base a techo por un paquete de $40 \mathrm{~cm}$ de areniscas marrones con un lag basal microconglomerático-bioclástico formado por extraclastos silícicos, intraclastos pelíticos de coloraciones marrones, bioclastos fragmentados atribuibles a ostreidos y gasterópodos y algunos nódulos ferruginosos limonitizados. A unos $20 \mathrm{~cm}$ por encima del lag basal se encuentra un nivel formado por moldes externos ferruginizados de troncos así como por fragmentos de troncos carbonizados asociados a bioclastos rodados. Los troncos tienen hasta $40 \mathrm{~cm}$ de longitud y en su superficie se localizan, en forma de molde interno las perforaciones estudiadas en el presente trabajo. Asociados a las perforaciones se encuentran restos fragmentarios y rodados de ostreidos (Ceratostreon tuberculiferum Koch \& Dunker, 1837), otros bivalvos indeterminados y gasterópodos (Gymnentome Cossman, 1909). También se han localizado restos de huesos de dinosaurio rodados con ostreidos incrustantes en su superficie, así como cutículas vegetales atribuibles a Frenelopsis ugnaensis Gómez, 2002. Sobre el nivel de areniscas aparecen 2,5 metros de facies heterolíticas en las que se aprecia cierta ritmicidad de niveles arenosos con ripples encima de los cuales se desarrollan finas pasadas carbonosas interestratificadas con arcillas limolíticas de color grisáceo finamente laminadas. Los interestratificados de arcillas-limolíticas y carbón tienen potencias milimétricas. Los niveles con ripples tienen bases planas y techos ondulados e internamente muestran laminaciones cruzadas planares. La potencia de estos niveles no sobrepasa los 5 $\mathrm{cm}$. Los niveles arenosos se pierden lateralmente pasando a arcillas limolíticas grisáceas.

En la zona de la vecina cantera del Mas de la Parreta, Teredolites ha sido reconocido también en una posición estratigráfica similar (Salas et al., 2003).

\section{DESCRIPCIÓN E ICNOTAXONOMÍA}

Las perforaciones reconocidas en los troncos de la Formación Arcillas de Morella se caracterizan por presentar una morfología en forma de botella con una apertura es- trecha que se ensancha progresivamente para acabar en una terminación semiesférica. La sección transversal es circular mientras que la morfología general puede ser recta o fuertemente contorsionada. Estas características permiten asignar las perforaciones al icnogénero Teredolites Leymerie, 1842. Este icnogénero es morfológicamente similar a Gastrochaenolites Leymerie, 1842, pero ambos se diferencian por las características del substrato en el que se desarrollan. Gastrochaenolites es propio de substratos minerales (líticos o esqueléticos) mientras que Teredolites se restringe a perforaciones en substratos de madera (xílicos). Esta diferenciación icnotaxonómica basada en las características del substrato fue ya propuesta por Leymerie (1842), aunque posteriormente fue criticada por Bradshaw (1980) que propuso el uso de Teredolites para todas las perforaciones en forma de botella. Sin embargo, Kelly \& Bromley (1984) en un trabajo de revisión de este tipo de estructuras de bioerosión recuperaron el significado original de ambos icnotaxones pues consideraron que desde el punto de vista del organismo perforante las diferentes características del substrato implicaban importantes diferencias ecológicas y construccionales. Esta propuesta es hoy ampliamente aceptada y ambos icnogéneros son de uso común en la literatura icnológica (e.g., Martinell, 1989).

Los ejemplares de Teredolites estudiados presentan dos morfologías diferentes. La más abundante corresponde a la icnoespecie Teredolites clavatus Leymerie, 1842. (Fig. 4 b-4 d). Ésta se caracteriza por tener un eje recto dispuesto perpendicularmente a la superficie del tronco y a las fibras de la madera. La sección longitudinal tiene forma de botella mientras que la sección basal es circular o elíptica. El diámetro de la perforación aumenta con la profundidad desde la superficie del tronco. Las perforaciones muestran un diámetro máximo de $10 \mathrm{~mm}$ y una longitud de hasta $15 \mathrm{~mm}$. Los ejemplares estudiados presentan relaciones longitud/anchura entre 1 y 1,5. Según Kelly \& Bromley (1984) el valor de este índice para esta especie es usualmente menor de 5. Algunas de las muestras presentan un alto grado de empaquetamiento de las perforaciones mientras que en otras éstas están más espaciadas. En las muestras con alto grado de perforación se observan perforaciones de varias dimensiones, con formas más desarrolladas que adquieren dimensiones mayores, entre las cuales se desarrollan ejemplares de menor tamaño aprovechando el espacio disponible. En ningún caso las perforaciones se cortan unas a otras.

La segunda morfología corresponde a la icnoespecie Teredolites longissimus Kelly \& Bromley, 1984, que los mismos autores describen como Teredolites de gran lon-

Figura 3. a, Columna estratigráfica de la Formación Arcillas de Morella en la Teuleria Milian (escala en metros). b, Detalle de los niveles superiores de la Formación Arcillas de Morella en donde se han localizado los troncos con Teredolites Leymerie, 1842.

$\boldsymbol{a}$, Stratigraphic section of the Morella Mudstone Formation at Teuleria Milian (scale in meters). $\boldsymbol{b}$, Close-up of the upper part of the formation where the logs with Teredolites Leymerie, 1842 are found. 
gitud con desarrollo sinuoso predominantemente paralelo a las fibras de los subtratos xílicos en donde se encuentra según su eje longitudinal. Estos Teredolites son más largos que la otra icnoespecie. En el material estudiado se caracteriza por presentar morfologías meandriformes con el eje longitudinal paralelo a las fibras de la madera. El índice longitud/anchura puede superar valores de hasta 8 (Fig. 4 a). Esta icnoespecie es menos abundante que $T$. clavatus en Teuleria Milian.

Ambas icnoespecies se conservan en forma de moldes internos con relleno pasivo de arena de granulometría media bastante micácea. La pared externa es en general lisa, no reconociéndose ni bioglifos (resultado de la actividad perforante del organismo) ni xenoglifos (que registran la estructura fibrosa del substrato xílico). En ningún caso se han observado revestimientos carbonatados (que pueden estar presentes en este icnogénero según Kelly \& Bromley, 1984), ni tampoco restos esqueléticos del posible productor. Esta ausencia puede ser debida a su disolución durante la diagénesis. Savrda et al. (1993) propusieron un esquema de clasificación de los modos de conservación de Teredolites. El material aquí descrito correspondería al tipo denominado relict log-ground caracterizado por la conservación únicamente de vestigios de madera encontrándose las perforaciones bien expuestas en forma de molde. El grado de conservación de los troncos es variable encontrándose en forma de impresiones (moldes externos) o bien carbonizados. Las perforaciones de Teredolites se localizan únicamente en los moldes de los troncos y no se han reconocido en los troncos carbonizados.

\section{PALEOBIOLOGÍA}

Teredolites, así como Gastrochaenolites, corresponde a perforaciones producidas por bivalvos (Kelly \& Bromley, 1984). El hábito perforante es bien conocido en diversos grupos de bivalvos, principalmente en las familias Gastrochaenidae, Pholadidae y Teredinidae pero también en diversos géneros de otras familias (Fischer, 1990; Savazzi, 1999). El mecanismo de bioerosión puede ser quími- co (por disolución, restringido a substratos carbonatados), mecánico (por rotación de la concha) o mixto (Savazzi, 1999). Las perforaciones en madera son producidas mecánicamente y actualmente las producen los Teredinidae y algunas formas de Pholadidae. En el registro fósil se han identificado ejemplos de Teredolites en cuyo interior se conservan las valvas del organismo productor (e.g., Kelly \& Bromley, 1984; Kelly, 1988), en cuyo caso se han observado especies de esas mismas familias. Kelly \& Bromley (1984) establecieron la similitud de Teredolites clavatus con las perforaciones del pholádido actual Martesia Sowerby, 1824, así como las de T. longissimus con las del teredínido Teredo Linné, 1758. Los grupos de bivalvos perforadores de madera actuales habitan medios marinos, aunque algunos Pholadidae pueden sobrevivir en medios salobres (Savazzi, 1999). Los substratos en los que pueden habitar son troncos flotantes, raíces de manglares, cáscaras de semillas y, en la actualidad, pilares de muelles o cascos de barcos (Kelly \& Bromley, 1984).

El icnogénero Teredolites se conoce desde el Pliensbaquiense (Jurásico Inferior), aunque los fósiles de representantes de las familias de bivalvos perforantes en madera no se encuentran hasta el Jurásico Medio (Kelly, 1988). A partir del Cretácico Teredolites es un icnogénero bastante común (e.g., Kelly, 1988; Mikuláš et al., 1995).

\section{IMPLICACIONES ESTRATIGRÁFICAS}

Teredolites es un icnogénero típicamente marino (Gingras et al., 2004). Solo se conoce un registro no marino del icnogénero en el Eoceno medio de Inglaterra (Plint \& Pickerill, 1985). Los autores citan la presencia de Teredolites en depósitos de lignito y en troncos que sitúan en un contexto fluvial. El significado de este material es poco claro; Plint \& Pickerill (1985) consideran que las perforaciones fueron producidas por algún grupo de bivalvos de agua dulce desconocido. Sin embargo, y dado que se trata de depósitos fluviales cercanos a la costa nos inclinamos a pensar que podrían estar reflejando cortas incursiones marinas en ambientes fluviales. A pesar de este registro y

Figura 4. Fotografías de los ejemplares de Teredolites Leymerie, 1842 (cada cuadro de la escala corresponde a $1 \mathrm{~cm}$ ). a, Ejemplar de Teredolites longissimus Kelly \& Bromley, 1984 con desarrollo paralelo a las fibras del tronco. b, Varios ejemplares de diferentes dimensiones de Teredolites clavatus Leymerie, 1842. c, Teredolites clavatus Leymerie, 1842 (izquierda) asociados a un molde de tronco (derecha) y a un ostreido (derecha inferior). d, Varios Teredolites clavatus Leymerie, 1842 en un tronco, destaca el desarrollo de las perforaciones perpendicular a las paredes del tronco y las perforaciones de diversos tamaños (ejemplar procedente de Mas de la Parreta, colección de Paleobotánica del Departament d'Estratigrafia, Paleontologia y Geociències Marines de la Universitat de Barcelona).

Photographs of specimens of Teredolites Leymerie, 1842 (each division in the scale is one centimeter). a, Teredolites longissimus Kelly \& Bromley, 1984 parallel to the log. b. Several specimens of different sizes of Teredolites clavatus Leymerie, 1842. c, Teredolites clavatus Leymerie, 1842 (left) associated to the mould of a log (right) and one oyster (right below). d, Several Teredolites clavatus Leymerie, 1842 in a log; their development perpendicular to the log surface and the coexistence of different sizes can be observed (specimen from Mas de la Parreta hosted in the Paleobotany collection of the Departament d'Estratigrafia, Paleontologia i Geociències Marines de la Universitat de Barcelona). 


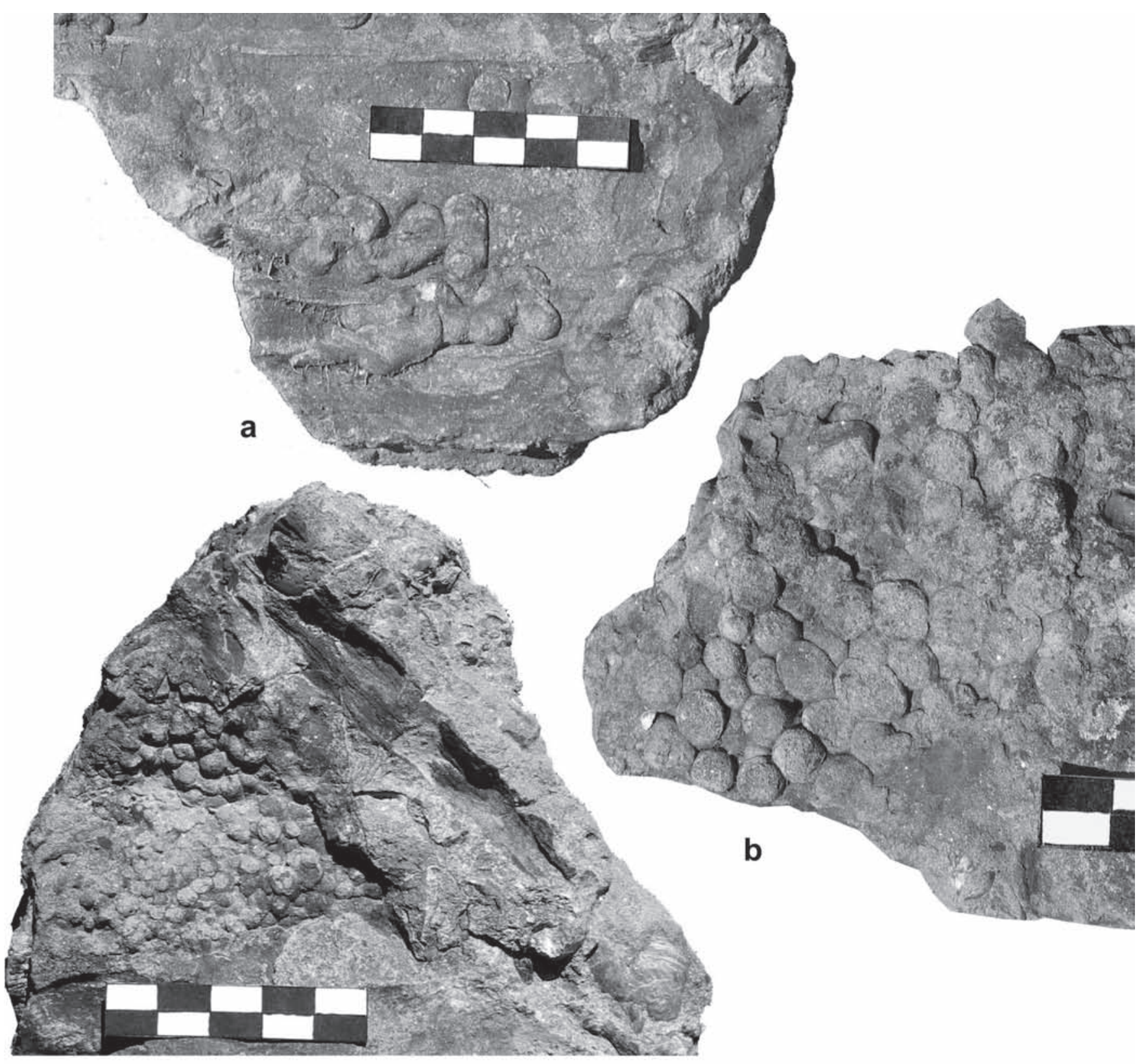

C

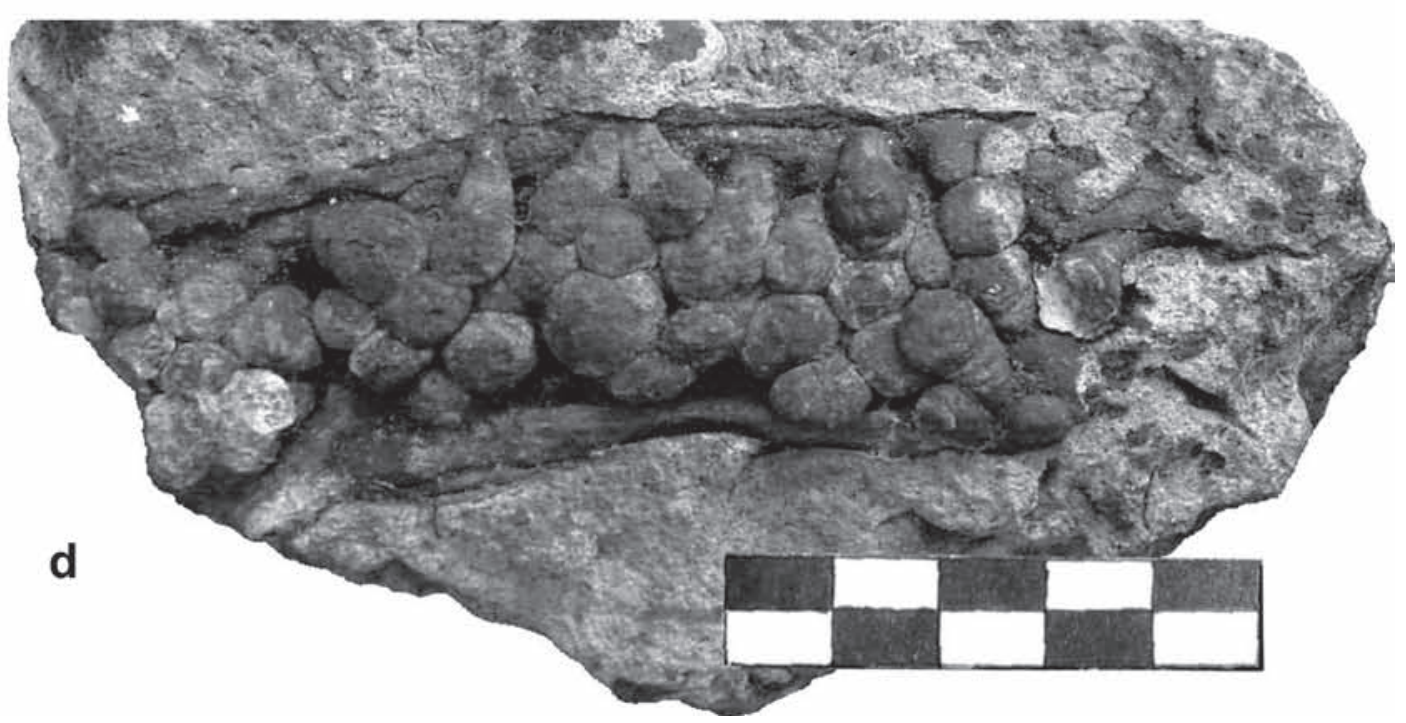


con prudencia podemos establecer que Teredolites es un indicador de influencia marina.

En el caso que nos ocupa hay que tener en cuenta que las perforaciones estudiadas aparecen en troncos transportados y acumulados. La bioerosión tuvo lugar previamente a la sedimentación de los troncos, cuando éstos se hallaban flotando en el mar como indica el hecho de que las perforaciones se encuentran distribuidas por toda la superficie del tronco. Esta aloctonía no permite asignar la asociación a la Icnofacies de Teredolites, puesto que en su definición original (Bromley et al., 1984) corresponde a perforaciones no transportadas en un substrato carbonoso formado por acumulación de restos vegetales.

A lo largo de toda la sección de la Teuleria Milian se reconocen tres niveles de acumulación de troncos. Sin embargo, en sólo uno de ellos se ha reconocido la existencia de Teredolites. La presencia de este icnogénero en los niveles más altos de la Formación Arcillas de Morella junto con las facies y estructuras sedimentarias allí encontradas confirma que estos depósitos tienen ya una clara influencia marina, probablemente mareal. Además, en los lags basales de los diversos canales se encuentran fragmentos de huesos de dinosaurios con organismos incrustantes marinos, así como ostreidos retrabajados. Así pues, el nivel en el que aparecen los troncos perforados marca el paso desde unas condiciones claramente continentales a otras con mayor influencia marina. La base de dicho nivel corresponde por tanto a una superficie transgresiva que separa los depósitos continentales correspondientes al cortejo de bajo nivel del mar (arcillas rojas) de los del cortejo transgresivo que corresponderían a una llanura siliciclástica mareal con desarrollo de barreras, con playas y canales mareales (Gámez et al., 2003).

La abundancia de troncos con Teredolites asociada a eventos transgresivos ha sido puesta de manifiesto por diversos autores (Savrda, 1991, 1995; Savrda et al., 1993; Tewari et al., 1998). Savrda (1991) y Savrda et al. (1993) interpretan esta situación como resultado de tres factores: 1) existencia de un mayor aporte de substratos xílicos en ambientes marinos y marinos marginales debido a la inundación de llanuras costeras forestadas; 2) retrabajamiento y concentración de substratos xílicos desarraigados durante la progresiva migración hacia continente de las playas (shorefaces); y 3) concentración de maderas flotantes en niveles de condensación en plataformas marinas. Dadas las características sedimentológicas del afloramiento estudiado, el primer escenario es el que mejor se adecua a los $\mathrm{Te}$ redolites de la Teuleria Milian. La presencia del nivel con Teredolites en la cantera del Mas de la Parreta en la misma posición estratigráfica (Salas et al., 2004), es indicadora de su persistencia lateral, y pone manifiesto su relación con el inicio del episodio transgresivo de la secuencia de depósito K 1.8 (Aptiense Inferior) y es, por tanto, de gran ayuda para la correlación de ambas series.

\section{CONCLUSIONES}

El estudio de la Formación Arcillas de Morella ha permitido reconocer la presencia de perforaciones en troncos asignables al icnogénero Teredolites. Se han identificado dos icnoespecies, T. clavatus y $T$. longissimus, que pueden ser atribuidas a la actividad de bivalvos perforantes, probablemente de las familias Pholadidae y Teredinidae, respectivamente.

Las perforaciones son alóctonas en el sentido de que fueron producidas sobre troncos flotantes previamente a su sedimentación. Por tanto, la asociación no puede ser identificada con la Icnofacies de Teredolites. Sin embargo, la presencia de Teredolites, junto con otros datos paleontológicos y sedimentológicos, pone de manifiesto la influencia marina en los depósitos que los contienen, y se constituye así en un marcador de un episodio transgresivo de la secuencia de depósito Aptiense Inferior. La identificación de un nivel de Teredolites en la cantera del Mas de la Parreta permite la correlación con la Teuleria Milian y evidencia la extensión lateral de los depósitos con troncos perforados.

\section{AGRADECIMIENTOS}

Los autores del presente trabajo agradecen al Dr. Ramón Salas el acceso al trabajo de Gámez et al. (2003) que en el momento de escribir el artículo se encontraba en prensa. También quisiéramos agradecer los comentarios del Dr. Gasulla sobre la estratigrafía y paleontología del Mas de la Parreta. De la misma forma extendemos los agradecimientos al Dr. Carles MartínClosas por proporcionar el ejemplar de la figura $4 \mathrm{~d}$ y por sus comentarios sobre los fósiles de vegetales. Las aportaciones de los revisores, Dra. Ana Rosa Soria y Dr. Eduardo Mayoral, han contribuido a mejorar la versión final de este artículo.

\section{BIBLIOGRAFÍA}

Beltrán, F. 1918. Fósiles de Morella. Boletín de la Real Sociedad Española de Historia Natural, 18, 134.

Bradshaw, M.A. 1980. Boring bivalves in the New Zealand Cenozoic with a redefinition of Teredolites. Records of the Canterbury Museum, 9, 289-294.

Bromley, R.G., Pemberton, S.G. \& Rahmani, R.A. 1984. A Cretaceous woodground: the Teredolites ichnofacies. Journal of Paleontology, 58, 488-498.

Canerot, J., Cungny, P., Pardo, G., Salas, R. \& Villena, J. 1982. Ibérica Central-Maestrazgo. In: El Cretácico de España. Universidad Complutense de Madrid, 273-344.

Fischer, R. 1990. Significado paleoecológico y geológico de perforaciones fósiles de bivalvos. Revista de la Sociedad Mexicana de Paleontología, 3, 79-95.

Gámez, D., Paciotti, P., Colombo, F. \& Salas, R. 2003. La Formación arcillas de Morella Aptiense inferior), Cadena 
Ibérica oriental (España): caracterización sedimentológica. Geogaceta, 34, 191-194.

Gingras, M. K., MacEachern, J.A. \& Pickerill, R.K. 2004. Modern perspectives on the Teredolites Ichnofacies: observations from Willapa Bay, Washington. Palaios, 19, 79-88.

Instituto Geológico y Minero de España. 1973. Mapa geológico de España. E. 1:50.000. Morella (545). $1^{\mathrm{a}}$ ed. Servicio de Publicaciones del Ministerio de Industria, Madrid.

Kelly, S.R.A. 1988. Cretaceous wood-boring bivalves from western antartica with a review of the Mesozoic Pholadidae. Palaeontology, 31, 341-372.

Kelly, S.R.A. \& Bromley, R.G. 1984. Ichnological nomenclature of clavate borings. Palaeontology, 27, 793-807.

Leymerie, M.A. 1842. Suite de mémoire sur le terrain Crétacé du département de l'Aube. Memoires de la Société Géologique de la France, 4, 291-364.

Martinell, J. 1989. Interacción organismos/sustrato duro: la bioerosión y sus implicaciones. In: Paleontología. Nuevas Tendencias (coord E. Aguirre). CSIC, Madrid, 205-222.

Mikuláš, R., Pek, I. \& Zimak, J. 1995. Teredolites clavatus from the Cenomanian near Maletin (Bohemian Cretaceous Basin), Moravia, Czech Republic. Věstník Ćeskeho Geologického Ústavu, 70, 51-57.

Plint, A.G. \& Pickerill, R.K. 1985. Non-marine Teredolites from the Middle Eocene of southern England. Lethaia, 18, 341-347.

Ruiz Omeñaca, J.I. \& Santos Cubedo, A. 1998. Un húmero de iguanodóntido (Ornitischia: Ornithopoda) del Cretácico inferior (Aptiense) de Morella (Castellón, España). Geogaceta, 24, 279-282.

Salas, R. \& Guimerà, J. 1996. Rasgos estructurales principales de la cuenca cretácica inferior del Maestrazgo (Cordillera Ibérica oriental). Geogaceta, 20, 1704-1706.

Salas, R. \& Guimerà, J. 1997. Estructura y estratigrafía secuencial de la cuenca del Maestrazgo durante la etapa de Rift Jurásica Superior-Cretácica Inferior (Cordillera Ibérica oriental). Boletín Geológico y Minero, 108, 65-74.

Salas, R. \& Martín-Closas, C. 1995. El Cretácico inferior del Nordeste de Iberia. In: Guia de Campo de las excursiones realizadas durante el III Coloquio del Cretácico de España. Morella, 1991. Publicacions Universitat de Barcelona, Barcelona, $153 \mathrm{pp}$.
Salas, R., Guimerà, J., Martín-Closas, C., Meléndez, A. \& Alonso, A. 2001. Evolution of the Mesozoic central Iberian Rift System and its Cainozoic inversion (Iberian chain). In: Peri-Tethyan Rift/Wrench Basins and Passive Margins (Eds. P.A. Ziegler, W. Cavazza, A.H.F. Robertson \& S. Crasquin-Soleau). Mémoires du Museum nant Histoire Naturelle, 186, 145-185.

Salas, R., Colombo, F., Gámez, D., Gómez, B., Gasulla, J.M., Martín-Closas, C., Moratalla, J., Paciotti, P., Querol, X. \& Solé, N. 2003. XIX Jornadas de la Sociedad Española de Paleontología. Guía de la excursión. Morella, 31 pp.

Santafé Llopis, J.V., Casanovas Cladellas, M.L., Sanz García, J.L. \& Calzada, S. 1978. Los Dinosaurios de Morella (nota preliminar). Acta geológica hispánica, 13, 149-154.

Santafé Llopis, J.V., Casanovas Cladellas, M.L., Sanz García, J.L. \& Calzada, S. 1981. Un nuevo yacimiento de dinosaurios en el Aptiense inferior de Morella (Castellón). Acta geológica hispánica, 16, 139-143.

Santafé Llopis, J.V., Casanovas Cladellas, M.L., Sanz García, J.L. \& Calzada, S. 1982. Geología y Paleontología (Dinosaurios) de las Capas Rojas de Morella (Castellón, España). Diputación Provincial de Castellón y Diputación de Barcelona, 15-60.

Savazzi, E. 1999. Boring, nestling and tube-dwelling bivalves. In: Functional morphology of the invertebrate skeleton (Ed. E. Savazzi). Wiley, Chichester, 209-237.

Savrda, C.E. 1991. Teredolites, wood substrates, and sea-level dynamics. Geology, 19, 905-908.

Savrda, C.E. 1995. Ichnologic applications in paleoceanographic, paleoclimatic, and sea-level studies. Palaios, 10, 565-577.

Savrda, C.E., Olazas, K., Demko, T.H., Huchison, R.A. \& Scheiwe, T.D. 1993 Log-grounds and the Ichnofossil Teredolites in Transgressive Deposit of the Clayton Formation (Lower Paleocene), Western Alabama. Palaios, 8, 311-324.

Tewari, A., Hart, M.B. \& Watkinson, M.P. 1998. Teredolites from the Garudamangalam Danstone Formation (late Turonian- Coniacian), Cauvery Basin, southeast India. Ichnos, 6, 75-98.

Manuscrito recibido: 1 de Diciembre, 2003 Manuscrito aceptado: 17 de Diciembre, 2004 